\title{
Characterizing Postural Stability in a Quasi-Static Sitting Position among Individuals with Sensorimotor Impairments Following Spinal Cord Injury
}

\author{
Murielle Grangeon ${ }^{1,2}$, Dany Gagnon ${ }^{1,2^{*}}$, Cyril Duclos ${ }^{1,2}$, Cindy Gauthier $^{1,2}$, Christian Larivière $^{1,3}$ and Philippe Gourdou ${ }^{2}$ \\ ${ }^{1}$ School of Rehabilitation, University of Montreal, Montreal, Canada \\ ${ }^{2}$ Pathokinesiology Laboratory, Centre for Interdisciplinary Research in Rehabilitation of Greater Rehabilitation Institute of Montreal Gingras-Lindsay, \\ Montreal, Canada \\ ${ }^{3}$ Robert-Sauvé Occupational Health and Safety Research Institute (IRSST), Montreal, Canada
}

\begin{abstract}
The objective of this study was to determine a minimum data set of postural measures to characterize seated stability in individuals with spinal cord injury $(\mathrm{SCl})$ by computing 39 Center-Of-Pressure (COP) measures routinely investigated in standing posture. Two short-sitting positions on an instrumented seat with the feet resting on force plates were compared between 14 individuals with $\mathrm{SCl}$ and 14 healthy controls: 1) with both hands on their thighs and 2) with both upper extremities flexed at $70^{\circ}$ and abducted at $45^{\circ}$. The correlations between all COP measures for the resultant, anteroposterior and mediolateral components were also computed. Differences in seated stability were observed between individuals with $\mathrm{SCl}$ and healthy controls, irrespective of the tasks. More precisely, the bilateral hand support was confirmed to be an effective strategy to compensate for anterior instability in individuals with SCl. As anticipated, time domain distance and frequency domain measures revealed complementary information. Distance and area COP measures were highly correlated with each other (i.e., redundant information) but were not correlated with frequency and hybrid measures. For both groups (between-task comparisons), the most discriminative uncorrelated measures were related to frequency parameters (i.e., independent information). Overall, our analyses revealed that a minimal data set of postural measures should include mean distance, mean velocity, centroidal frequency, median power frequency and frequency dispersion. These measures should be reported for all directional components whenever applicable, as both anteroposterior and mediolateral activities independently contribute to the resultant COP outcome measures.
\end{abstract}

Keywords: Postural control; Rehabilitation; Spinal cord injury; Sitting balance; Center of pressure

Abbreviations: SCI: Spinal Cord Injury; U/E: Upper Extremities; COM: Center of Mass; BOS: Base of Support; COP: Center of Pressure; RD: Resultant Direction; AP: Anteroposterior; ML: Mediolateral; AIS: American Impairment Scale

\section{Introduction}

Individuals with a Spinal Cord Injury (SCI) often experience various degrees of motor or sensory impairments that can affect their trunk as well as their Upper Extremities (U/Es) and lower extremities depending on the neurological level and the completeness of the injury to the spinal cord (i.e., impaired sensorimotor pathways originating in the spinal cord). Thus, the synergies required to regulate quasistatic sitting postural stability are affected among individuals with SCI [1]. Moreover, the rapid sublesional bone mineral density loss [2], in addition to muscle hypertrophy that frequently develops at the U/Es (except in individuals with high SCI who generally develop hypotrophy at the U/Es), raises the height of the center of mass (COM) (i.e., the relative body mass in individuals with SCI was more in the upper body compared to able-bodied individuals). Unfortunately, this may further jeopardize the ability to secure quasi-static sitting postural stability following SCI which may, in turn, affect the ability to perform certain functional activities in daily life, especially activities that solicit the U/ Es [3].

Postural stability can be defined as the ability to steadily maintain the vertical projection of the COM of the body within the Base of Support (BOS) [4]. In a sitting position, the BOS is restricted by the supporting area encompassing the buttocks, thighs and feet, whereas the COM is expected to be located approximately in front of the $4^{\text {th }}$ lumbar vertebra and above the ischial tuberosities. Postural steadiness is often characterized using the displacement of the center of pressure
(COP) over time, which reflects the location of the vertical reaction force vector on an instrumented surface (i.e., force plate) on which a person stands or sits [5]. Facilitated by coordinated neuromuscular response [6], the COP excursion, confined within the BOS, is aligned in such a way that it continuously regulates the COM to prevent postural instability [7]. Postural outcome measures commonly used to quantify postural stability include time and frequency domain parameters to measure the displacement, velocity, area and frequency characteristics of COP fluctuations over time [8-11]. Some studies have also performed comparisons to identify the most relevant measures to differentiate between various groups or tasks, and most of them have investigated standing position [12-16]. Prieto et al. [15] proposed 39 COP-related outcome measures linked to time and frequency domains to investigate age-related change in standing postural stability. These measures, particularly COP mean velocity, successfully confirmed agerelated change in standing postural stability between young able-bodied and elderly adults, under both eyes-open and eyes-closed conditions. Interestingly, Rocchi et al. [16] investigated the amount of redundancy

*Corresponding author: Dany Gagnon, Pathokinesiology Laboratory, Centre for Interdisciplinary Research in Rehabilitation of Greater Rehabilitation Institute of Montreal Gingras-Lindsay, 6300 Avenue Darlington, Montreal, QC, Canada, Tel: +1-514-343-6111; E-mail: dany.gagnon.2@umontreal.ca

Received January 22, 2013; Accepted March 08, 2013; Published March 12 2013

Citation: Grangeon M, Gagnon D, Duclos C, Gauthier C, Larivière C, et al. (2013) Characterizing Postural Stability in a Quasi-Static Sitting Position among Individuals with Sensorimotor Impairments Following Spinal Cord Injury. J Bioengineer \& Biomedical Sci 3: 124. doi:10.4172/2155-9538.1000124

Copyright: (c) 2013 Grangeon M, et al. This is an open-access article distributed under the terms of the Creative Commons Attribution License, which permits unrestricted use, distribution, and reproduction in any medium, provided the original author and source are credited. 
Citation: Grangeon M, Gagnon D, Duclos C, Gauthier C, Larivière C, et al. (2013) Characterizing Postural Stability in a Quasi-Static Sitting Position among Individuals with Sensorimotor Impairments Following Spinal Cord Injury. J Bioengineer \& Biomedical Sci 3: 124. doi:10.4172/21559538.1000124

Page 2 of 10

of these same outcome measures among young able-bodied individuals to select a minimal data set allowing for a straightforward interpretation of standing postural stability. They also evaluated the relative weight of Anteroposterior (AP) and Mediolateral (ML) directional components on the COP parameters analysis. They concluded that the mean velocity of both directional components should be analyzed to inform about regulatory activity in AP and ML directions. They also suggested that analysis of the distance and frequency measures in the AP direction can portray the COP dispersion and the spectral density of the COP path.

Unfortunately, quantitative assessment of seated postural stability in able-bodied individuals [17] and in various clinical populations [18], such as individuals with SCI $[19,20]$, has not received much attention to date. Van Dieën et al. [17] investigated the interrelations between 39 parameters characterizing COP movements in seated postural stability among able-bodied individuals and their relationships with seated instability (i.e., participants were not able to maintain a sitting position without U/E support and ultimately used the safety rail to control their sitting balance). Sway parameters were found to be strongly interrelated; nevertheless, no single variable could be related to loss of balance. Additionally, a wobble chair was employed and prevented their feet from making contact with the floor, which is not easily generalizable to seated postural tasks of individuals with SCI in daily life. Among individuals with SCI, biomechanical studies on quasi-static seated stability $[19,20]$ have investigated COP fluctuations in the AP direction only, with COP deviation, mean distance and mean velocity as main outcome measures. Despite previous studies that have highlighted the importance of considering COP frequency measures and those that have been extended to include investigations in the ML direction [21], such a biomechanical approach has not been yet analyzed in this population. Interpreting the results of postural stability assessment can be difficult to manage in clinical environments, particularly within SCI rehabilitation programs due to the large data set that can be extracted from the COP. A simple method that allows for straightforward interpretation is needed for quantifying seated postural stability in clinical populations.

The main objective of the present study was to compare the postural stability of individuals with SCI and able-bodied individuals using a biomechanical approach. Two quasi-static short-sitting positions mainly used in daily life among individuals with SCI were considered: sitting with both hands positioned on the thighs and sitting with both U/Es flexed and abducted. The secondary objective of this study was to determine a minimal data set that would be small enough to reduce computation and avoid redundancy, while being large enough to rigorously characterize quasi-static seated postural stability in individuals with SCI. To identify actual and meaningful differences in postural stability and adjustments between and within groups, COP outcome measures were investigated on the horizontal plane (Resultant Distance (RD)) and along the AP and ML directional components. It was hypothesized that seated postural stability in individuals with SCI would differ from that of able-bodied individuals, particularly when U/Es support was reduced. Indeed, hand support was expected to represent a compensatory strategy for supporting part of the body weight (arm-head-trunk segment) and for optimizing seated postural stability among individuals with SCI. Based on previous findings in ablebodied individuals [15], it was also expected that many COP-related outcome measures based on a time series analysis would provide highly redundant information. Finally, it was also anticipated that the ML information would provide meaningful postural indications on seated stability, complementary to the AP information, in individuals with SCI since sensorimotor impairment primarily affecting postural muscles are involved in the control of both AP and ML trunk displacements.

\section{Materials and Methods}

\section{Participants}

An experimental group of 14 individuals with SCI and a gender-, age-, height, and weight-matched control group of 14 able-bodied individuals volunteered to participate in this study after having met the inclusion criteria (Table 1). Individuals with SCI had sustained a complete or incomplete SCI (American Impairment Scale (AIS): A-D) affecting various spinal cord neurological levels (C3-T11) at least three months prior to the study. Individuals were eligible to participate in this study if they were able to independently maintain an unsupported short-sitting position with their feet resting on the floor for one minute, use a manual wheelchair as their primary mode of mobility

\begin{tabular}{|c|c|c|c|c|c|c|c|c|}
\hline \multicolumn{2}{|c|}{ Groups } & Age (year) & Height $(\mathrm{cm})$ & Weight (Kg) & $\begin{array}{l}\text { Time since injury } \\
\text { (year) }\end{array}$ & Level of injury & AIS score & $\begin{array}{l}\text { Base of support } \\
\text { area }\left(m^{2}\right)\end{array}$ \\
\hline \multirow{16}{*}{$\begin{array}{l}\text { Individuals with } \\
\mathrm{SCI}(\mathrm{N}=14)\end{array}$} & & 72 & 1.87 & 105.6 & 2.56 & T6 & D & 0.39 \\
\hline & & 23 & 1.68 & 86.3 & 0.88 & C3 & D & 0.33 \\
\hline & & 46 & 1.83 & 109.1 & 5.02 & L1 & C & 0.39 \\
\hline & & 49 & 1.73 & 84.5 & 1.73 & C5 & D & 0.37 \\
\hline & & 53 & 1.78 & 129.6 & 5.14 & T10 & A & 0.36 \\
\hline & & 44 & 1.70 & 73.8 & 2.67 & T10 & A & 0.27 \\
\hline & & 57 & 1.88 & 98.2 & 2.99 & T10 & B & 0.33 \\
\hline & & 26 & 1.63 & 46.8 & 2.78 & T11 & A & 0.25 \\
\hline & & 32 & 1.75 & 65.1 & 3.09 & $\mathrm{C} 7$ & B & 0.25 \\
\hline & & 25 & 1.80 & 52.9 & 2.77 & $\mathrm{~T} 4$ & A & 0.25 \\
\hline & & 30 & 1.88 & 98.1 & 3.33 & T10 & A & 0.34 \\
\hline & & 49 & 1.83 & 78.1 & 25.90 & $\mathrm{~T} 7$ & B & 0.31 \\
\hline & & 25 & 1.83 & 76.5 & 2.08 & C6 & A & 0.32 \\
\hline & & 40 & 1.70 & 59.3 & 0.15 & $\mathrm{C} 7$ & B & 0.24 \\
\hline & Mean & 41 & 1.78 & 83.1 & 4.36 & & & 0.31 \\
\hline & SD & 15 & 0.08 & 23.3 & 6.34 & & & 0.05 \\
\hline \multirow{2}{*}{$\begin{array}{l}\text { Able-bodied } \\
\text { individuals } \\
\quad(\mathrm{N}=14)\end{array}$} & Mean & 39 & 1.74 & 80.0 & & & & 0.32 \\
\hline & $\mathrm{SD}$ & 14 & 0.07 & 17.0 & & & & 0.05 \\
\hline \multicolumn{2}{|c|}{ Between-group comparisons } & $p=0.77$ & $p=0.25$ & $p=0.69$ & & & & $p=0.70$ \\
\hline
\end{tabular}


Citation: Grangeon M, Gagnon D, Duclos C, Gauthier C, Larivière C, et al. (2013) Characterizing Postural Stability in a Quasi-Static Sitting Position among Individuals with Sensorimotor Impairments Following Spinal Cord Injury. J Bioengineer \& Biomedical Sci 3: 124. doi:10.4172/21559538.1000124

for at least 4 hours per day (i.e., participants with AIS-D were not classified as community ambulators) and had an activity tolerance of at least 60 minutes, with rest and pressure-relief periods offered as needed. None of the able-bodied participants reported having a musculoskeletal impairment that affected their trunk, lower extremities or U/Es or any other condition that might have altered their ability to maintain a short-sitting position. The study was conducted at the Pathokinesiology Laboratory at the Institut de réadaptation GingrasLindsay-de-Montréal. Ethical approval was obtained from the Research Ethics Committee of the Centre for Interdisciplinary Research in Rehabilitation of Greater Montreal (CRIR-456-0809). All participants read and signed the informed consent form before initiating the study.

\section{Experimental tasks}

Participants were centrally positioned on a height-adjustable instrumented seat covered with a $2 \mathrm{~cm}$ thick high-density foam with their back unsupported and their feet resting on two force plates embedded in the floor. The effect of the cushion type on postural control is inconsistent in literature [22,23]. Therefore, different cushions were tested among individuals with SCI and able-bodied adults previously to the present study and no significant difference in COP measures were found. In the present study, participants sat with their thighs parallel to one another and with approximately $75 \%$ of the length of their thighs in contact with the seat. Their knees were flexed at approximately $85^{\circ}$, while their feet were parallel to one another on the floor. Participants were instructed to seat upright in a comfortable selfselected position and to randomly maintain two quiet sitting positions with both hands resting on their thighs (Task \#1: supported sitting) and with both $\mathrm{U} / \mathrm{Es}$ flexed at $70^{\circ}$ and horizontally abducted at $45^{\circ}$ (Task \#2: unsupported sitting). These two different $\mathrm{U} / \mathrm{E}$ positions aimed to isolate the compensatory role of the U/Es when maintaining a quiet sitting position. Additionally, these two sitting positions are considered usual in daily living and the abduction and flexion of shoulders are commonly used for reaching and grasping tasks (e.g., ecological validity). During these tasks, participants were asked to look at a target located three meters in front of them at eye level. One 60-second trial in each position was performed. A two-minute rest period, during which pressure-relief lifts or shifts were performed as needed, was offered between the two experimental tasks to minimize fatigue and to protect skin integrity at the buttocks. The physiotherapist supervised the participants closely to ensure optimal safety during both experimental tasks while one research associate coordinated the computerized data acquisition and storage during both trials. A familiarization session was also conducted within 7 days of the testing session.

\section{Instrumentation and data processing}

Kinetic data were recorded at a sampling frequency of $600 \mathrm{~Hz}$ using a height-adjustable instrumented seat (width $=53.5 \mathrm{~cm}$; depth=50.8 cm) composed of four force transducers directly underneath the left and right buttocks, coupled with two force plates embedded in the floor underneath the left and right feet (combined size: $w i d t h=92.6 \mathrm{~cm}$; depth $=50.8 \mathrm{~cm}$ ). These surfaces allowed for the continuous recording of ground reaction forces underneath the left and right buttocks as well as underneath the left and right feet needed to compute the local COP position at each instant at each force plate within their own local referential. The tri-axial components of these reaction forces (from the four contact points) corresponding to the anteroposterior $(\mathrm{Fx})$, vertical $(\mathrm{Fy})$ and mediolateral $(\mathrm{Fz})$ directions were then combined to compute the global 3D COP position over time within the laboratory system (Figure 1). In the horizontal plane, the coordinate of the global COP trajectory was computed using the

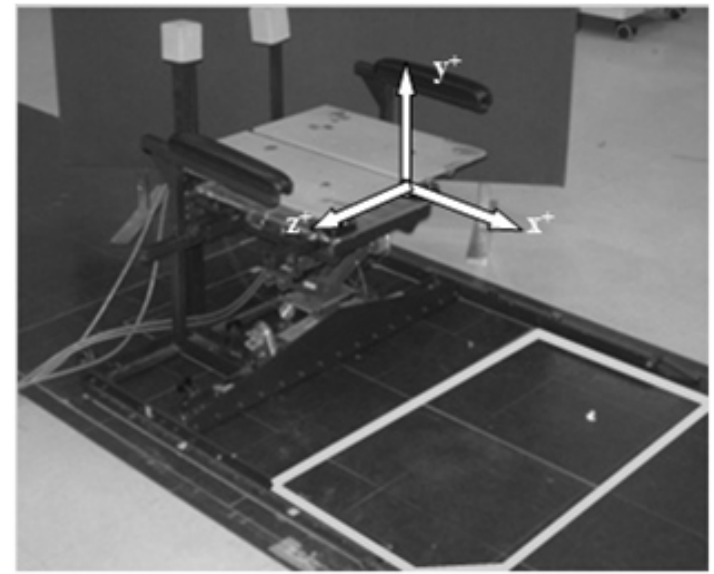

Figure 1: Instrumented seat and floor allowing for the continuous recording of reaction forces underneath the buttocks and feet during quiet sitting positions in the laboratory environment along with the coordinate system. Note that $2-\mathrm{cm}$ thick high density foam is added on the seat during the assessment.

Fx and Fz time series with an in-house $\mathrm{C}++$ program. The COP time series recorded were filtered with a fourth-order Butterworth zerolag low-pass digital filter with a cut-off frequency of $5 \mathrm{~Hz}$ and then down-sampled to $300 \mathrm{~Hz}$. Additional information pertaining to kinetics is available $[24,25]$

\section{COP-related outcome measures}

A 30 -second period (time elapsed between the $15^{\text {th }}$ and $45^{\text {th }}$ second for each recorded trial) was used to compute the outcome measures. A typical example of the stabilogram for an individual with SCI and for a control participant during the two tasks, from which outcome measures were extracted, is illustrated in figure 2 .

Quasi-static seated postural stability outcome measures were computed based on the procedures and equations proposed by Prieto et al. [15], and commonly used in studies investigating postural steadiness [14,26]. Specifically, a total of seventeen parameters representing several time- and frequency-domain COP outcome measures (hereafter simply referred to as "COP measures") were computed in the horizontal plane using the signals recorded during each trial (Table 2). Out of the seventeen COP measures, eleven were composed of AP (Fx time series) and $\mathrm{ML}$ (Fz time series) components from which the RD component was calculated at each data point. The remaining six COP measures were extracted from the RD time series in the horizontal plane only, because they described planar characteristics (e.g., the area covered by the COP).

In the present study, postural stability is represented by three commonly reported dimensions: stability performance, control demand and postural regulations (Table 2). Out of the seventeen COP measures, seven distance and area measures estimated the displacement of the COP (which was related to the stability performance) and the velocity of the COP (which was related to control demand [13,18,27]). An increase in control demand has often been associated with increased visual contribution for postural stability [28-30] and increased risk of falling [31]. Therefore, a decrease in stability performance (i.e., increase in COP displacement) associated with increased control demand (i.e., increased COP velocity) would be interpreted as reduced postural stability. Five "hybrid" measures modeled the stabilogram by combining the distance measures and velocity to quantify the relationship between 
Citation: Grangeon M, Gagnon D, Duclos C, Gauthier C, Larivière C, et al. (2013) Characterizing Postural Stability in a Quasi-Static Sitting Position among Individuals with Sensorimotor Impairments Following Spinal Cord Injury. J Bioengineer \& Biomedical Sci 3: 124. doi:10.4172/21559538.1000124

Page 4 of 10

\begin{tabular}{|c|c|c|c|}
\hline Types of measure & Outcome measures & Description & Interpretation \\
\hline \multirow[t]{5}{*}{ Time-domain distance measures } & $\operatorname{MDIST}(\mathrm{mm})^{\mathrm{a}}$ & Average distance from the mean COP & Indicators of stability performance \\
\hline & RDIST $(\mathrm{mm})^{\mathrm{a}}$ & RMS distance & \\
\hline & TOTEX $(\mathrm{mm})^{\mathrm{a}}$ & Total length of the COP path & \\
\hline & RANGE $(\mathrm{mm})^{\mathrm{a}}$ & Maximum distance between any two points & \\
\hline & $\operatorname{MVELO}(\mathrm{mm} / \mathrm{s})^{\mathrm{a}}$ & Average velocity of the COP & Indicators of control demand \\
\hline \multirow[t]{2}{*}{ Time-domain area measures } & AREA-CC $\left(\mathrm{mm}^{2}\right)$ & $95 \%$ confidence circle area & Indicators of stability performance \\
\hline & AREA-CE $\left(\mathrm{mm}^{2}\right)$ & 95\% confidence ellipse area & \\
\hline \multirow[t]{5}{*}{ Time-domain hybrid measures } & AREA-SW (mm²/s) & Sway area & $\begin{array}{l}\text { Indicators of the relationship between control demand and } \\
\text { stability performance }\end{array}$ \\
\hline & MFREQ $(\mathrm{Hz})^{\mathrm{a}}$ & Mean frequency & \\
\hline & FD-RD & Fractal dimension & \\
\hline & FD-CC & Fractal dimension based on the area-CC & \\
\hline & FD-CE & Fractal dimension based on the area-CE & \\
\hline \multirow[t]{5}{*}{ Frequency domain measures } & T POWER ${ }^{a}$ & Total power frequency & Indicators of postural regulations \\
\hline & $50 \%(\mathrm{~Hz})^{\mathrm{a}}$ & Median power frequency & \\
\hline & $95 \%(\mathrm{~Hz})^{\mathrm{a}}$ & $95 \%$ power frequency & \\
\hline & CFREQ $(\mathrm{Hz})^{\mathrm{a}}$ & Centroidal frequency & \\
\hline & FREQDa & Frequency dispersion & Indicators of trunk stiffness \\
\hline
\end{tabular}

aComputed based on resultant distance (RD) time series as well as anteroposterior (AP) and mediolateral (ML) time series.

Table 2: Summary of the COP-related outcome measures.

control demand and stability performance. The frequency distribution of the COP displacement was calculated for the frequency range from 0.15 to $5 \mathrm{~Hz}$ to define the shape of the power spectral density. Five frequency COP measures provided information on postural regulations. Three power frequency measures often indicate change in preferential postural regulation $[32,33]$. The centroidal frequency often documents the inertia of an inverted pendulum [26] and the time for a system to return to its initial position. It is acknowledged that in a sitting position, the trunk most likely behaves like an inverted pendulum system rotating at the hip joints. Thus, trunk muscle impairments among individuals with SCI may increase trunk inertia and increase the time for the COP to return to the initial position, thereby demonstrating lower oscillation frequency compared to able-bodied individuals. Lastly, frequency dispersion is a unit-less measure of variability in frequency content. It may also be related to active and/or passive stiffness or rigidity of the trunk [14].

\section{Statistical analysis}

Descriptive statistics were calculated for all participant characteristics and for all COP measures in both groups. After the normality of these data had been verified with Kolmogorov-Smirnov tests, a two-way ANOVA for independent samples with one between factor [individuals with SCI versus healthy counterparts] and one within factor [supported versus unsupported sitting] was performed for each quasi-static outcome measure to verify if differences existed across groups, tasks or both. If any interaction was revealed $(\mathrm{p}<0.05)$, $\mathrm{t}$-tests with Bonferroni adjustment were applied $(\mathrm{p}<0.5 / 2)$. If no interaction was revealed, a group or/and task effect (i.e., main effect) would be analyzed.

Pearson's product-moment correlation coefficients ( $r$ ) were also computed to quantify the strength of the association between all COP outcome measures obtained for the supported and unsupported sitting position using the merged data from the two groups in each component direction. Measures with a very strong correlation $(\mathrm{r} \geq$ 0.90) were pooled together to form strongly correlated COP measure subgroups since they were deemed to provide the same information. The strongest correlated variable in each correlation subgroup was then selected as the most representative measure of this subgroup. Measures with a weak correlation $(r<0.70)$ were considered to provide independent information. Each of these uncorrelated measures had to be sufficiently discriminative between groups and between tasks (i.e., results of the ANOVA analyses) to be selected. Then, the minimum data set defined from this selection process was deemed sufficient to provide a comprehensive assessment of quasi-static seated postural stability in a short-sitting position in individuals with SCI. Lastly, correlation coefficients were also used to verify the specific influence of AP and ML components on outcome measures obtained in the RD component. All statistical analyses were performed using SYSTAT $^{\circ} \mathrm{v} 9.0$ software for Windows.

\section{Results}

All COP measures calculated during supported and unsupported sitting obtained for individuals with SCI and able-bodied individuals are summarized in table 3, as well as between- and within-group comparisons. Only the AREA-CC, TPOWER-RD and TPOWER-AP were found not to be significantly different, irrespective of the group or task.

\section{Between-group comparisons}

CFREQ and 95\% power frequency measures were the only COP measures to be significantly different between groups among all directional components, irrespective of the task. During the supported sitting position, most of the between-group differences were revealed by the hybrid (6/7) and frequency (13/15) measures. Among the distance measures, only MDIST, RDIST and RANGE significantly differed between groups, particularly in terms of the ML directional component. Among the area measures, no significant difference was found between the groups. During unsupported sitting, all COP measures differed at least for one directional component between groups. Among the distance and area measures, TOTEX and MVELO both for RD and AP, as well as AREA-SW, significantly differed between groups during unsupported sitting compared to supported sitting.

In terms of significant differences among the distance and hybrid measures, the individuals with SCI had greater values than the ablebodied individuals, irrespective of the task. Conversely, all frequency measures were found to be significantly different, aside from dispersion frequency, the individuals with SCI obtained lower values than the able- 
Citation: Grangeon M, Gagnon D, Duclos C, Gauthier C, Larivière C, et al. (2013) Characterizing Postural Stability in a Quasi-Static Sitting Position among Individuals with Sensorimotor Impairments Following Spinal Cord Injury. J Bioengineer \& Biomedical Sci 3: 124. doi:10.4172/21559538.1000124

Page 5 of 10

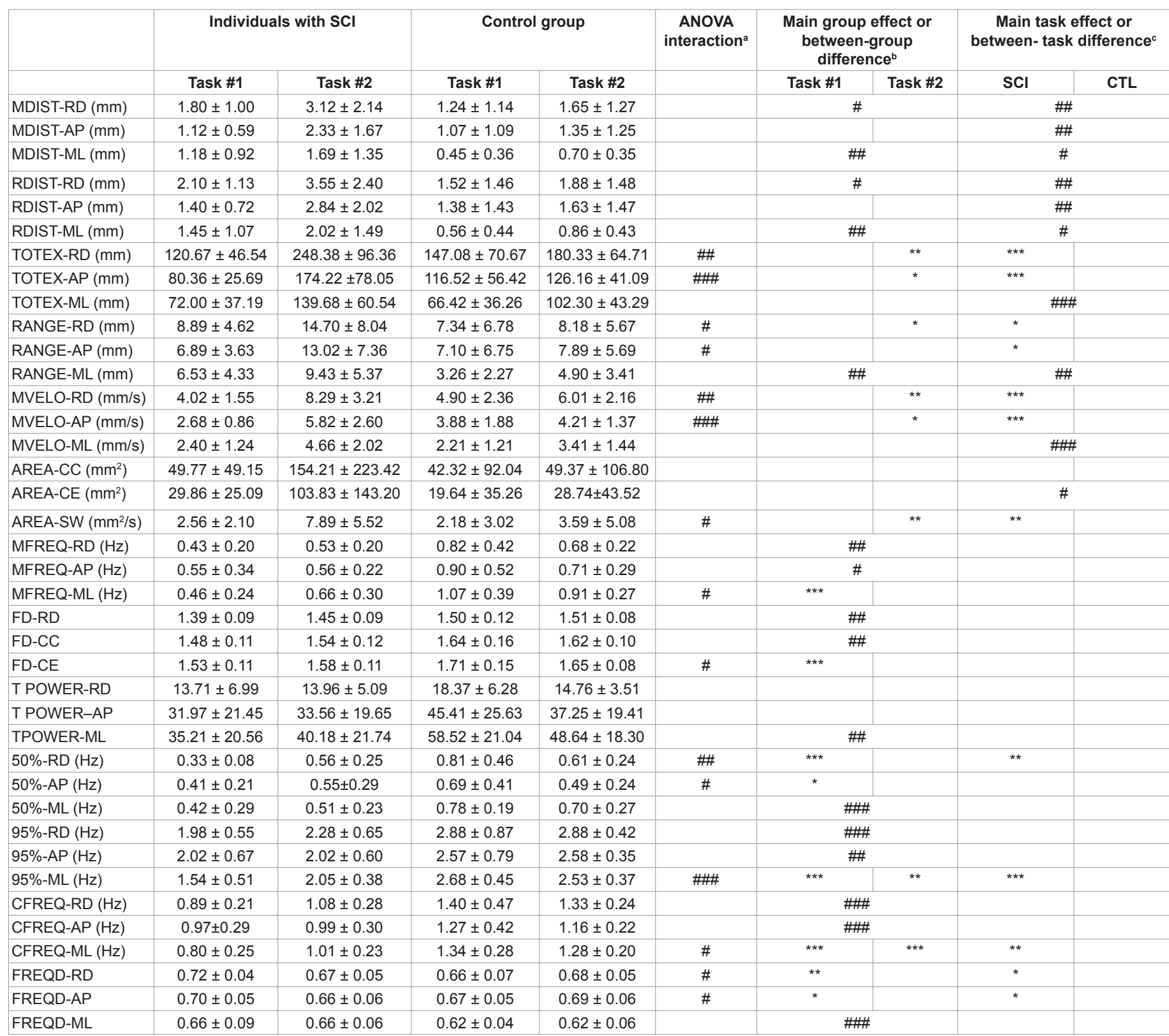

a ANOVA interaction results. ${ }^{b}$ Main group effect or t-tests between groups when ANOVA interaction was significant in the supported (Task \#1) or unsupported sitting position (Task \#2). ${ }^{c}$ Main task effect or t-tests between tasks within each group when ANOVA interaction was significant. For interaction and main effect, \#= $p<0.05$; \#\#= $p$ $\leq 0.01$; \#\#= $\leq \leq 0.001$. For t-tests with Bonferroni adjustments, ${ }^{*}=p<0.025 ;{ }^{* *}=p \leq 0.01 ;{ }^{* * *} p \leq 0.001$. SCl: individuals with SCl; CTL: control group.

Table 3: Mean \pm standard deviations of COP measures and statistical results.

bodied individuals, regardless of the position. The dispersion frequency was greater in the individuals with SCI compared to the able-bodied individuals during supported sitting for RD, AP and ML components and during unsupported sitting for the ML component. Finally, stronger statistically significant between-group differences $(\mathrm{p}<0.001)$ were generally found among the hybrid and frequency measures in comparison to the distance and area measures.

\section{Between-task comparisons (supported and unsupported sitting)}

MDIST and RDIST were the only COP measures to be significantly different between tasks for all directional components, irrespective of the group. RANGE-ML, TOTEX-ML, MVELO-ML and AREA-CE were also found to increase during unsupported sitting compared to supporting sitting, irrespective of the group. Additionally, in individuals with SCI, significant task differences were found for RANGE, TOTEX, MVELO and FREQD for both RD and AP components. AREA-SW was also found to be significantly different between tasks in this group, as well as 50\%-RD, 95\%-ML and CFREQ-ML. All these measures increased during the unsupported sitting position compared to the supported sitting position, except for FREQD which decreased. Interestingly, stronger between-task statistical differences were obtained among individuals with SCI $(7$ measures with $p<0.001)$ than among the ablebodied individuals $(2$ measures with $\mathrm{p}<0.001)$.

\section{Cross-correlations between COP measures}

The measures for which very strong correlations were found for each 
Citation: Grangeon M, Gagnon D, Duclos C, Gauthier C, Larivière C, et al. (2013) Characterizing Postural Stability in a Quasi-Static Sitting Position among Individuals with Sensorimotor Impairments Following Spinal Cord Injury. J Bioengineer \& Biomedical Sci 3: 124. doi:10.4172/21559538.1000124

component (RD, AP and ML) during both unsupported and supported sitting were merged together and allowed for 4 correlation subgroups for the RD component, and 5 correlation subgroups for the AP and ML components (Figure 3). Interestingly, no correlation was found between the distance and frequency measures. The hybrid measures were either correlated with distance or frequency measures. Mean distance and mean velocity were the strongest correlated variables among the correlation subgroups for distance and area measures, irrespective of the directional component. Among the correlation groups for frequency and hybrid measures, centroidal frequency and AREASW measures were the strongest correlated variables, irrespective of the directional component. The 8 measures considered to be weakly correlated with the others were linked to frequency measures. More precisely, frequency dispersion and total power frequency measures were the weakest correlated measures for the three components, as well as the $50 \%$ power frequency measures for the $\mathrm{AP}$ and $\mathrm{ML}$ directional components.

For the 11 measures with RD, AP and ML components, the information obtained for the RD component was more influenced by the AP component $(r=0.92 \pm 0.10$ and $r=0.83 \pm 0.19$ for supported and unsupported sitting, respectively) than the ML component ( $r=0.68$ \pm 0.17 and $r=0.63 \pm 0.29$ for supported and unsupported sitting, respectively) in the able-bodied individuals, regardless of the task ( $p<0.001$ and $p=0.01$ in supported and unsupported sitting respectively). The correlation coefficients were stronger during supported sitting than unsupported sitting in this group, irrespective of the directional component $(p=0.02)$. In individuals with SCI, the information obtained for the RD component was equally influenced by the AP $(r=0.73 \pm 0.21)$ and ML $(r=0.69 \pm 0.30)$ components during supported sitting $(p=0.36)$.
During unsupported sitting, the information obtained for the RD component was more influenced by the AP component $(r=0.89 \pm 0.08)$ than the ML $(r=0.79 \pm 0.10)$ component $(p=0.008)$. The correlation coefficients were weaker during supported sitting than unsupported sitting, regardless of the directional component $(p=0.002)$. In both groups, the correlation between the $\mathrm{AP}$ and $\mathrm{ML}$ components was weaker than the correlations between the resultant and AP or ML component, irrespective of the task.

\section{Discussion}

\section{Between-group comparisons}

In the present study, it was elected to quantify postural stability using three commonly reported dimensions: stability performance, control demand and postural regulations. As previously explained in the method section and in table 2, these dimensions were quantified using distinct COP outcome measures as previously described in the literature $[14,15]$ although no universal consensus yet exists with regard to the interpretation of these measures and their clinical meaningfulness $[18,21]$. The choice of adhering to these three commonly reported dimensions was also motivated by the fact that one of the objective of the present study was to select a minimal data set to be used in clinical practice by physical and occupational therapists while assessing quasistatic sitting stability among individuals with SCI.

As expected, the COP measures based on distance and frequency analyses revealed between-group differences in postural stability while maintaining a quasi-static sitting position. More precisely, during supported sitting, individuals with SCI exhibited lower ML stability performance (i.e., increased distance measures) than the able-bodied
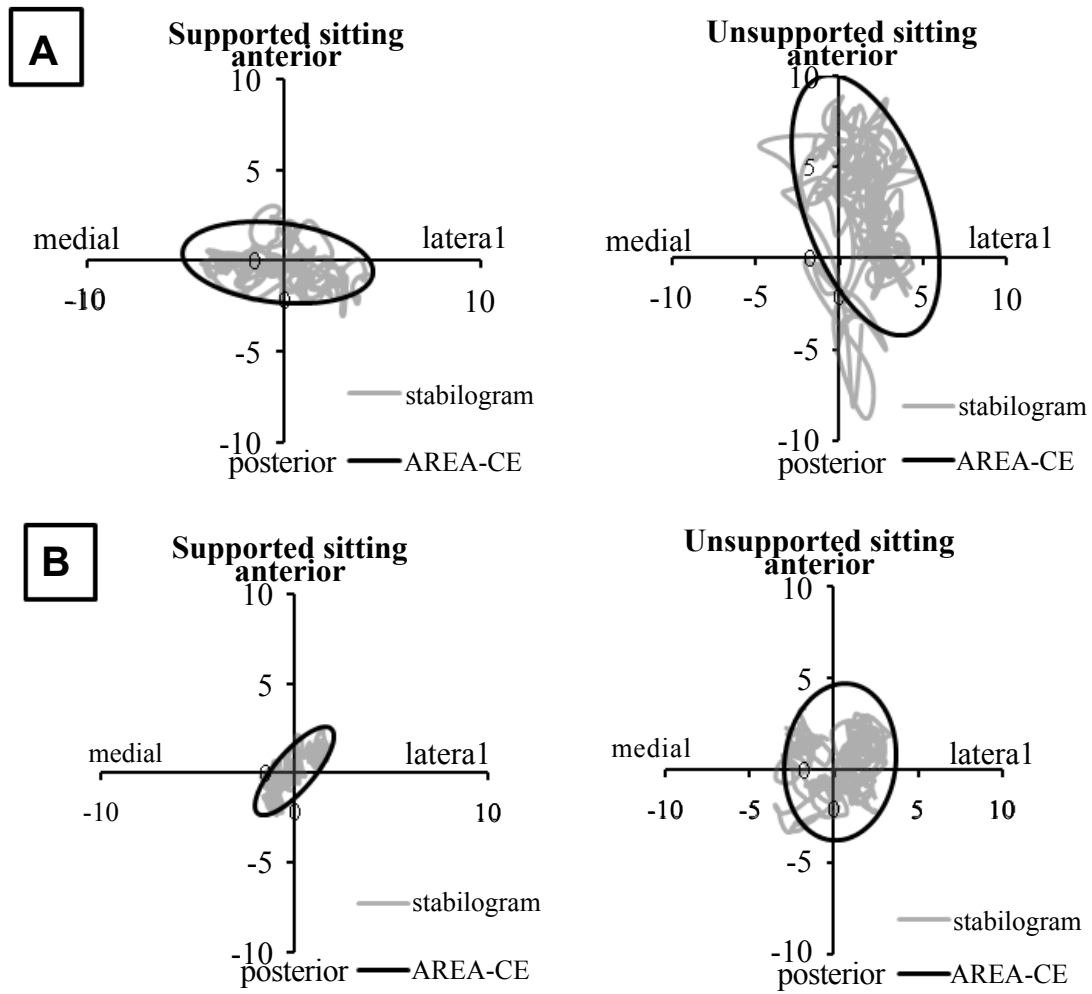

Figure 2: Representative stabilogram for a participant with a complete sensorimotor spinal cord injury at the 10th thoracic vertebra and for a healthy control (B) based on sitting postures. A 95\% confidence ellipse area (AREA-CE) ( $\mathrm{mm} 2$ ) estimating the confidence area of the COP path on the force plate that encloses approximately $95 \%$ of the points on the COP path, is illustrated 
Citation: Grangeon M, Gagnon D, Duclos C, Gauthier C, Larivière C, et al. (2013) Characterizing Postural Stability in a Quasi-Static Sitting Position among Individuals with Sensorimotor Impairments Following Spinal Cord Injury. J Bioengineer \& Biomedical Sci 3: 124. doi:10.4172/21559538.1000124

Page 7 of 10

individuals with no difference in control demand (i.e., mean velocity) even though different postural regulations (i.e., frequency measures) were observed. Additionally, greater frequency variability (i.e., frequency dispersion measures) observed in individuals with SCI compared to able-bodied individuals might reflect the muscular stochastic activity regulating ML stability due to altered sensory afferences and muscular synergies [14,34]. These results are consistent with those of Shirado et al. [20], who found a predominantly ML trajectory pattern of the COP in individuals with SCI compared to an AP pattern in the control group during a long-sitting position with the legs extended and the hands on the thighs. Two non-mutually exclusive explanations are possible. Firstly, the reduced force generating capability of left and right obliquus abdominis externi and interni, as well as of the bilateral low back and hip muscles, might provide incomplete muscular compensations to control lateral trunk motions. Secondly, the height of the COM that raises proximally (cephalically) in the trunk compared to able-bodied individuals might increase active and passive muscle contributions for postural adjustments [35], particularly in the ML direction as shown in the present study. For the AP component, the shift in the trunk-head segment mass might be counteracted by the bilateral hand support, limiting increased postural demand and regulations for this directional component. Indeed, the AP instability revealed among individuals with SCI during unsupported sitting compared to supported sitting, confirmed that U/E support might be an alternative strategy to compensate for AP instability and back and hip extensor motor impairments [36].

During unsupported sitting, ML instability increased in both groups as well as AP instability in individuals with SCI, compared to supported sitting. Compared to able-bodied participants, individuals with SCI still exhibited reduced ML stability performance despite relatively similar AP stability performance (i.e., COP distance measures). Nevertheless, a longer path of the COP was revealed in individuals with SCI compared to able-bodied individuals, particularly for the AP directional component. These results coincide with those of Shirado et al. [20], who found an increase in the COP path during long sitting with both arms stretched out over the thighs in individuals with SCI compared to able-bodied individuals. Shirado et al. [20] also found a similar shape of the COP trace (i.e., central pattern) in both groups with no difference in COP distance for the AP component. The U/Es maintained over the thighs or forward (i.e., both shoulders flexed at $70^{\circ}$ and abducted at $45^{\circ}$ ) in combination with the anterior foot support might help stabilize the sitting position and reduced differences in AP COP displacement between groups. This reduced difference in AP COP displacement might also be due to the neurological level of individuals included in the present study. It is plausible that individuals with low SCI (partial or total use of abdominal/low back muscles) reach values comparable to those obtained among healthy participants as recently documented when evaluating the multidirectional limits of stability in sitting among individuals with SCI [37]. It may explain why no difference occurred on the AP directional component between individuals with SCI and ablebodied individuals, since almost $50 \%$ of the group has a SCI at or below the $10^{\text {th }}$ thoracic AIS neurological level.

Additionally, a greater AP control demand was observed in individuals with SCI compared to supported sitting. This result might be explained by increased integration of visual information [30] to control seated stability in individuals with SCI when the U/Es are restricted

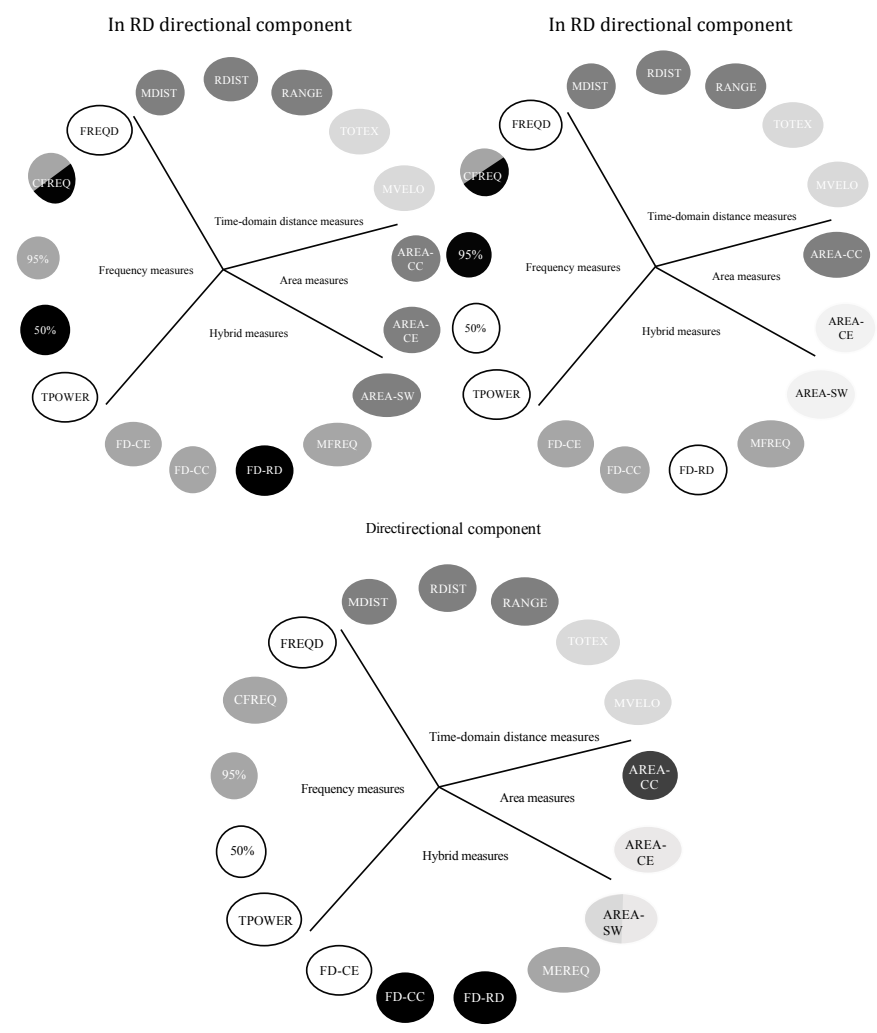

Figure 2: Relationships between COP measures during both positions for both groups in each directional component. Each correlation subgroups ( $r \geq 0.90)$ appeared in a distinct grey color. Weakly correlated measures $(r<0.70)$ are illustrated in white. 
due to altered proprioceptive feedback from the postural muscles. Secondly, decreased AP frequency variability also found in individuals with SCI compared to supported sitting suggested the additional use of muscular synergies to control AP stability during unsupported sitting, particularly by the non-postural muscles [38-40]. The time since the initial SCI was sustained might have impacted this result. Previous studies showed that individuals with the most long-lasting SCI tended to exhibit increased control demand on the AP directional component with no altered effect on stability performance, irrespective of the sitting position. These results suggested a preferential and optimal use of non-postural muscles as a result of time and learning [41]. Based on electromyography studies among individuals with SCI [40], the co-activation of thoracohumeral muscles (e.g., latissimus dorsi and trapezius pars ascendens), the upper thoracic portion of the erector spinae and the scapular protractors (e.g., pectoralis major and serratus anterior) might provide posterior stabilization controlling anterior displacements of the trunk segment [39]. Nevertheless, further studies ideally incorporating electromyography investigations of postural (i.e., trunk) and non-postural muscles should be done to assess these hypotheses.

To summarize, reduced ML stability performance associated with a change in ML postural regulations was revealed in individuals with SCI compared to able-bodied individuals, irrespective of the level of difficulty imposed by the two sitting tasks, thereby suggesting compensatory postural strategies with regard to the ML component to stabilize posture. The unsupported sitting position also evidenced decreased AP stability performance and differences in AP postural control in individuals with SCI. Therefore, assessing these individuals without the use of their U/Es to support part of their body weight appears to be more informative than in supported sitting when evaluating seated stability ability.

\section{Selection of minimal data set to quantify postural steadiness}

The significant differences found between the two tasks and between groups were closely related to the correlation subgroups. This information is relevant for researchers and clinicians when selecting postural measures that might provide complementary information. Computing more than one measure from any correlation subgroup may be redundant. As expected, distance and area COP measures were found to be strongly correlated with each other. Nevertheless, distance $\mathrm{COP}$ measures were found to be more group- and task-discriminative than area COP measures. They highlighted significantly reduced ML stability in individuals with SCI compared to able-bodied individuals, irrespective of the tasks. Stronger between-task statistical differences were found among distance measures compared to area measures. Despite all these measures that characterize the size of the stabilogram, distance measures represent the COP as it evolves along both the AP and ML directional components, whereas area measures represent the COP evolution on the entire horizontal plane. Thus, distance measures may be more influenced by biomechanical factors such as the BOS than area measures [42]. This therefore explains the results reported in table 3. However, some authors have normalized the COP measures relative to the BOS area, foot length [43] and other anthropometric parameters. No consensus has yet been reached on the normalization approach for assessing sitting postures. Given that no between-group difference was found for the area of the BOS, height, or body mass of participants in this study, the outcome measures were not normalized. Furthermore, AP and ML measures can be more indicative of the true directional components of the sway when foot placement is constrained to a position aligned with axes of the force plates as in the present study [44]. Constraining the foot position indeed minimizes cross-talk between AP and ML information, which may occur if the anatomical frame of the subject is not precisely aligned with the reference frame of the platform.

Additionally, choosing at least one measure from each domain proposed by Prieto et al. [15] (Table 2) to define all aspects of seated postural stability appears indicated. In the few articles focusing on seated stability in individuals with SCI $[19,20,22]$, distance, velocity and area $\mathrm{COP}$ measures were mainly represented, whereas frequency $\mathrm{COP}$ measures were not investigated. However, the present results confirm that frequency measures provide complementary and meaningful information [26] for describing differences in postural regulation, particularly between groups and between tasks for AP component among individuals with SCI. These measures did not provide discriminative information among able-bodied individuals, probably due to the fact that quite similar postural regulation might be expected among this group.

Finally, according to the comparison and correlation results obtained in the present study, the following minimal data set of COP measures is suggested when quantifying seated stability among individuals with SCI: Mean Distance (MDIST), Mean Velocity (MVELO), Centroidal Frequency (CFREQ), Median Power Frequency (50\%) and Frequency Dispersion (FREQD). These measures should be reported for all directional components whenever applicable, as both $\mathrm{AP}$ and $\mathrm{ML}$ components modulate the RD component in individuals with SCI. Furthermore, partitioning the movements along the ML and AP axes provides an additional opportunity to assess the effect of the impairment of the thoracohumeral and trunk muscles, since muscles predominantly acting in the AP synergy can be dissociated from those involved in the ML synergy. This minimum data set is almost identical to that proposed by Prieto et al. [15] and Rocchi et al. [21], who also investigated the redundancy of COP measures. However, the population investigated (i.e., elderly individuals and individuals with Parkinson's disease, respectively) that the task (i.e., standing posture) and the analysis tools used (i.e., multidimensional selection procedure based on the principal component analysis by Rocchi et al. [21]) were not identical and might explain the few differences in the selected COP measures. Of course, since the minimal data set of COP measures established in the present study remains population-specific, they may not be generalizable to other populations (e.g., neurologic disease, orthopedic disease) who experience sitting postural instability. Nevertheless, among individuals who experience sensorimotor impairments similar to those experienced by individuals with SCI, it is anticipated that the present minimal data set of COP measures may be appropriate to define quasi-static seated postural stability. Furthermore, even among individuals with SCI, additional investigations should be done to evaluate the reliability of the selected COP measures and to further clarify whether the minimal data set proposed can sufficiently discriminate among postural stability exhibited by individuals with SCI.

\section{Study limitations}

Although this study is relevant for researchers and clinicians who wish to quantify seated postural stability in individuals with SCI, there are some limitations. The small sample of individuals with SCI and able-bodied individuals in this study warrants consideration. Moreover, the limited number of individuals with SCI who participated in the study did not allow us to form subgroups of participants based on their sensorimotor impairments. Factors such ASIA motor and sensory scores, completeness of the SCI and time since SCI investigated in a larger sample size could strengthen the results and provide 
Citation: Grangeon M, Gagnon D, Duclos C, Gauthier C, Larivière C, et al. (2013) Characterizing Postural Stability in a Quasi-Static Sitting Position among Individuals with Sensorimotor Impairments Following Spinal Cord Injury. J Bioengineer \& Biomedical Sci 3: 124. doi:10.4172/21559538.1000124

Page 9 of 10

relevant information in the future. Since individuals with a high SCI (i.e., tetraplegia) may not be able to maintain a sitting position with both arms flexed and abducted for 60 seconds or may quickly become fatigued, intermediate positions should also be investigated in the future. Increasing the number and/or time of trials would have restricted the participation of individuals with severe impairment in the present study.

From a biomechanical perspective, when individuals maintain a short-sitting position with their feet firmly positioned on the floor, the lower limbs support a significant proportion of the body weight as previously documented [24]. For this reason, it would have been interesting to analyze the changes in COP outcome measures resulting from a reduction, even elimination of foot support (unsupported short-sitting position), with both hands positioned on the thighs and with both U/Es flexed and abducted. Additionally, the use of complement postural measures to confirm the analysis of postural regulations based on frequency analysis should be done. Indeed, this analysis could be impacted by which frequencies were used (i.e. frequency range between 0.15 and $0.5 \mathrm{~Hz}$ ). Although the results of other studies using different frequencies [18] did not find frequencies below $0.15 \mathrm{~Hz}$, the choice of the lowest frequency in function of the trial duration for data analysis (i.e. 30s) may be discussed. Duplicating the frequency analysis of Prieto et al. [15] quantifying standing postural stability may not be applicable when focusing on sitting postural stability. However, in a recent study comparing sitting and standing positions [26], the frequency measures during quiet sitting were consistently larger than during quiet standing. Therefore, any event that could appear below $0.15 \mathrm{~Hz}$ was supposed to not provide information about postural control system. However, other methods such as the stabilogram diffusion function (SDF) measures developed by Collins and de Luca [45] may be used in the future to refine the present investigation. This approach may allow one to define two control schemes: an open-loop control scheme implying no neural control over short time intervals, and a closed-loop control scheme implying the presence of neural feedback control over longer time intervals.

\section{Conclusion}

As expected, the individuals with SCI exhibited different seated postural stability, irrespective of the tasks compared to the healthy controls. The use of the U/Es to provide additional support to the trunk segment was thus found to be an effective compensatory strategy for optimizing seated postural stability over time in individuals with SCI. Finally, the present study confirmed that the use of COP measures usually allocated to the study of standing balance may also be applied for investigating seated stability among individuals with SCI. The use of only a few distance and area measures (e.g., mean distance, RMS, range) may allow clinicians to rigorously assess seated postural stability given the high redundancy, whereas mean velocity and frequency measures (e.g., median power frequency, centroidal frequency, frequency dispersion) provide independent information. Therefore, the minimal data set suggested in the present study may be used by researchers when performing a comprehensive seated postural assessment among individuals with SCI and may be adjusted based on the information they wish to obtain. Finally, the present set-up may be appropriate to define quasi-static seated postural stability in other populations (e.g., neurologic disease, orthopedic disease) to define a population-specific minimal data set of COP measures.

\section{Acknowledgements}

This project was funded in part by the Quebec Rehabilitation Research Network (REPAR) and the Fonds de la recherche en santé du Québec (FRSQ). Murielle Grangeon holds a postdoctoral fellowship from the SensoriMotor Rehabilitation Research Team (SMRRT). Dany Gagnon holds a Junior 1 Research Career Award from the FRSQ. Cindy Gauthier was supported by a summer research studentship financed by the Quebec Rehabilitation Research Network (REPAR). Dany Gagnon and Cyril Duclos are members of the Multidisciplinary SensoriMotor Rehabilitation Research Team (www.errsm.ca) supported by the CIHR.

The equipment and material required for the research completed at the Pathokinesiology Laboratory was financed in part by the Canada Foundation for Innovation (CFI) and the Lindsay Rehabilitation Hospital Foundation. Specia thanks are expressed to Professor Sylvie Nadeau, PT, PhD, Michel Goyette, BSc Eng, Pierre Desjardins, MScA, Eng and Daniel Marineau, DEC for their intellectual, engineering and technical contributions that enriched this project.

\section{References}

1. Seelen HAM, Pottne YJM, Pons C (1999) Adaptation and relearning of postural control in thoracic spinal cord injured subjects. Biomedical Aspects of Manual Wheelchair Propulsion, Van Kemenade CHHM, van der Woulde LVH, (eds). The Netherlands IOS Press, Amsterdam, Netherlands 443: 208-216.

2. Garland DE, Adkins RH, Stewart CA (2012) Bone Impairment and Spinal Cord Injury. In: Stone JH, Blouin M, (eds.), International Encyclopedia of Rehabilitation.

3. Sprigle S, Wootten M, Sawacha Z, Thielman G (2003) Relationships among cushion type, backrest height, seated posture, and reach of wheelchair users with spinal cord injury. J Spinal Cord Med 26: 236-243.

4. Horak FB (1987) Clinical measurement of postural control in adults. Phys Ther 67: $1881-1885$.

5. Winter DA (1995) A.B.C. (Anatomy, Biomechanics and Control) of Balance during Standing and Walking. Waterloo Biomechanics.

6. Massion J (1994) Postural control system. Curr Opin Neurobiol 4: 877-887.

7. Winter DA, Patla AE, Prince F, Ishac M, Gielo-Perczak K (1998) Stiffness control of balance in quiet standing. J Neurophysiol 80: 1211-1221.

8. Capodaglio P, Cimolin V, Vismara L, Grugni G, Parisio C, et al. (2011) Postural adaptations to long-term training in Prader-Willi patients. J Neuroeng Rehabil 8: 26.

9. Cimolin V, Galli M, Grugni G, Vismara L, Precilios H, et al. (2011) Postural strategies in Prader-Willi and Down syndrome patients. Res Dev Disabil 32: 669-673.

10. Galli M, Cimolin V, Vismara L, Grugni G, Camerota F, et al. (2011) The effects of muscle hypotonia and weakness on balance: a study on Prader-Willi and Ehlers-Danlos syndrome patients. Res Dev Disabil 32: 1117-1121.

11. de Haart M, Geurts AC, Huidekoper SC, Fasotti L, van Limbeek J (2004) Recovery of standing balance in postacute stroke patients: a rehabilitation cohort study. Arch Phys Med Rehabil 85: 886-895.

12. Baratto L, Morasso PG, Re C, Spada G (2002) A new look at posturographic analysis in the clinical context: sway-density versus other parameterization techniques. Motor Control 6: 246-270.

13. Hufschmidt A, Dichgans J, Mauritz KH, Hufschmidt M (1980) Some methods and parameters of body sway quantification and their neurological applications. Arch Psychiatr Nervenkr 228: 135-150.

14. Maurer C, Peterka RJ (2005) A new interpretation of spontaneous sway measures based on a simple model of human postural control. J Neurophysiol 93: $189-200$.

15. Prieto TE, Myklebust JB, Hoffmann RG, Lovett EG, Myklebust BM (1996) Measures of postural steadiness: differences between healthy young and elderly adults. IEEE Trans Biomed Eng 43: 956-966.

16. Rocchi L, Chiari L, Cappello A (2004) Feature selection of stabilometric parameters based on principal component analysis. Med Biol Eng Comput 42 71-79.

17. van Dieën JH, Koppes LL, Twisk JW (2010) Postural sway parameters in seated balancing; their reliability and relationship with balancing performance. Gait Posture 31: 42-46.

18. Genthon N, Vuillerme N, Monnet JP, Petit C, Rougier P (2007) Biomechanical 
Citation: Grangeon M, Gagnon D, Duclos C, Gauthier C, Larivière C, et al. (2013) Characterizing Postural Stability in a Quasi-Static Sitting Position among Individuals with Sensorimotor Impairments Following Spinal Cord Injury. J Bioengineer \& Biomedical Sci 3: 124. doi:10.4172/21559538.1000124

assessment of the sitting posture maintenance in patients with stroke. Clin Biomech (Bristol, Avon) 22: 1024-1029.

19. Chen CL, Yeung KT, Bih LI, Wang CH, Chen MI, et al. (2003) The relationship between sitting stability and functional performance in patients with paraplegia. Arch Phys Med Rehabil 84: 1276-1281.

20. Shirado O, Kawase M, Minami A, Strax TE (2004) Quantitative evaluation of long sitting in paraplegic patients with spinal cord injury. Arch Phys Med Rehabil 85: $1251-1256$.

21. Rocchi L, Chiari L, Cappello A, Horak FB (2006) Identification of distinct characteristics of postural sway in Parkinson's disease: a feature selection procedure based on principal component analysis. Neurosci Lett 394: 140-145.

22. Aissaoui R, Boucher C, Bourbonnais D, Lacoste M, Dansereau J (2001) Effect of seat cushion on dynamic stability in sitting during a reaching task in wheelchair users with paraplegia. Arch Phys Med Rehabil 82: 274-281.

23. de Abreu DC, Takara K, Metring NL, Reis JG, Cliquet A Jr (2012) Interference of different types of seats on postural control system during a forward-reaching task in individuals with paraplegia. Int J Rehabil Res 35: 208-213.

24. Gagnon D, Nadeau S, Noreau L, Dehail P, Gravel D (2008) Quantification of reaction forces during sitting pivot transfers performed by individuals with spinal cord injury. J Rehabil Med 40: 468-476.

25. Nadeau S, Desjardins P, Brière A, Roy G, Gravel D (2008) A chair with a platform setup to measure the forces under each thigh when sitting, rising from a chair and sitting down. Med Biol Eng Comput 46: 299-306.

26. Vette AH, Masani K, Sin V, Popovic MR (2010) Posturographic measures in healthy young adults during quiet sitting in comparison with quiet standing. Med Eng Phys 32: 32-38.

27. Geurts AC, Nienhuis B, Mulder TW (1993) Intrasubject variability of selected force-platform parameters in the quantification of postural control. Arch Phys Med Rehabil 74: 1144-1150.

28. Fitzgerald JE, Murray A, Elliott C, Birchall JP (1994) Comparison of body sway analysis techniques. Assessment with subjects standing on a stable surface. Acta Otolaryngol 114: 115-119.

29. Elliott C, FitzGerald JE, Murray A (1998) Postural stability of normal subjects measured by sway magnetometry: pathlength and area for the age range 15 to 64 years. Physiol Meas 19: 103-109.

30. Cornilleau-Pérès V, Shabana N, Droulez J, Goh JC, Lee GS, et al. (2005) Measurement of the visual contribution to postural steadiness from the COP movement: methodology and reliability. Gait Posture 22: 96-106.

31. Piirtola M, Era P (2006) Force platform measurements as predictors of falls among older people - a review. Gerontology 52: 1-16.
32. Demura S, Kitabayashi T, Noda M (2008) Power spectrum characteristics of sway position and velocity of the center of pressure during static upright posture for healthy people. Percept Mot Skills 106: 307-316.

33. Norrlin S, Karlsson A, Ahlsten G, Lanshammar H, Silander HC, et al. (2002) Force measurements of postural sway and rapid arm lift in seated children with and without MMC. Clin Biomech (Bristol, Avon) 17: 197-202.

34. van der Burg JC, van Wegen EE, Rietberg MB, Kwakkel G, van Dieën JH (2006) Postural control of the trunk during unstable sitting in Parkinson's disease. Parkinsonism Relat Disord 12: 492-498.

35. Rosker J, Markovic G, Sarabon N (2011) Effects of vertical center of mass redistribution on body sway parameters during quiet standing. Gait Posture 33: $452-456$

36. Reeves NP, Cholewicki J, Narendra KS (2009) Effects of reflex delays on postural control during unstable seated balance. J Biomech 42: 164-170.

37. Gauthier C, Gagnon D, Grangeon M, Jacquemin G, Nadeau S, et al. (2012) Comparison of multidirectional seated postural stability between individuals with spinal cord injury and able-bodied individuals. J Rehabil Med.

38. Curtis KA, Kindlin CM, Reich KM, White DE (1995) Functional reach in wheelchair users: the effects of trunk and lower extremity stabilization. Arch Phys Med Rehabil 76: 360-367.

39. Seelen HA, Potten YJ, Huson A, Spaans F, Reulen JP (1997) Impaired balance control in paraplegic subjects. J Electromyogr Kinesiol 7: 149-160.

40. Seelen HA, Potten YJ, Drukker J, Reulen JP, Pons C (1998) Developmen of new muscle synergies in postural control in spinal cord injured subjects. $J$ Electromyogr Kinesiol 8: 23-34.

41. Grangeon M, Gagnon D, Gauthier C, Jacquemin G, Masani K, et al. (2012) Effects of upper limb positions and weight support roles on quasi-static seated postural stability in individuals with spinal cord injury. Gait Posture 36: 572-579.

42. Chiari L, Rocchi L, Cappello A (2002) Stabilometric parameters are affected by anthropometry and foot placement. Clin Biomech (Bristol, Avon) 17: 666-677.

43. Maki BE, Holliday PJ, Fernie GR (1990) Aging and postural control. A comparison of spontaneous- and induced-sway balance tests. J Am Geriatr Soc 38: 1-9.

44. Kapteyn TS, Bles W, Njiokiktjien CJ, Kodde L, Massen CH, et al. (1983) Standardization in platform stabilometry being a part of posturography. Agressologie 24: 321-326.

45. Collins JJ, De Luca CJ (1995) The effects of visual input on open-loop and closed-loop postural control mechanisms. Exp Brain Res 103: 151-163. 